\title{
EXCITONS AND PHONONS IN GaN. MAGNETOOPTICAL AND SPATIALLY RESOLVED INVESTIGATIONS
}

\author{
A. Hoffmann, L. Eckex, H. Siegle, A. Kaschner \\ Institut für Festkörperphysik, Technische Universität Berlin \\ Hardenbergstr. 36, 10623 Berlin, Germany
}

\section{J. Christen, F. Bertram}

Institut für Experimentelle Physik, Otto-von-Guericke-Universität Magdeburg PO4120, 39016 Magdeburg, Germany

AND Q.K.K. LIU

Hahn-Meitner Institut Berlin, Theoretische Physik

Glienicker Str. 100, 14109 Berlin, Germany

A comprehensive study of the direct photoluminescence from free-exciton states in $\mathrm{GaN}$ using polarization-dependent and magnetooptical measurements is presented. We measured and identified fine-structure splittings in the $n=1$ state of the $A$-exciton. From the magnetoluminescence data obtained in fields up to $15 \mathrm{~T}$ we determined the $g$-values of the conduction and valence bands parallel and perpendicular to the $c$-axis. Self-organized hexagonal GaN pyramids of $5 \mu \mathrm{m}$ width and covered by six $\{1 \underline{101}\}$ side facets were investigated by spatially resolved cathodoluminescence and micro-Raman spectroscopy. Beside a narrow luminescence peak at $355 \mathrm{~nm}$, originating from the $2 \mu \mathrm{m}$ thick GaN layer, an additional broad luminescence band was observed from the GaN pyramids around a wavelength of $357 \mathrm{~nm}$. A strong energy shift is found along the $\{1 \underline{101}\}$ pyramidal facets and directly visualized by monochromatic cathodoluminescence images and line scans. In GaN epilayers grown on $\mathrm{GaAs}$ substrates a series of sharp modes in the range between $60 \mathrm{~cm}^{-1}$ and $250 \mathrm{~cm}^{-1}$ for temperatures below $100 \mathrm{~K}$ was found. The intensities of these modes increased drastically with decreasing temperature. Raman excitation spectra showed a maximum between $514.5 \mathrm{~nm}$ and $568 \mathrm{~nm}$. A comparison of spatially resolved investigations with that of intentionally doped GaN epilayers showed that the in-diffusion of As from the substrate plays an important role. Raman spectra as a function of external fields, like magnetic field and hydrostatic pressure, gave additional information about the defect type and the underlying scattering mechanism.

PACS numbers: 71.35.-y, 78.40.Fy, 78.45.+h 


\section{Introduction}

Excitons play a key role in the investigation of basic optical and electronic properties of wide-band semiconductors. Rapid progress in this field in recent years notwithstanding the excitonic fine structure very much featured in fundamental research and discussions [1]. For many wide-gap semiconductors it was investigated by means of transmission and reflection spectroscopies. For the case of GaN reflection spectra have been published by many groups revealing mainly the energy differences between the three top valence bands and some fine structure due to higher excited states (e.g., [2]). Transmission spectroscopy in the excitonic range, however, could not reveal any fine structure, mostly because only light polarized perpendicular to the $c$-axis can be used for epitaxial layers. Much of the fine structure information on excitons in hexagonal wide-gap semiconductors was obtained from the "forbidden" polarization $\boldsymbol{E} \| \boldsymbol{c}$. To overcome this problem many-photon absorption has been used for GaN [3]. In the first part of the paper we show that carefully analyzed polarized photoluminescence spectra give a comprehensive access to the excitonic fine structure of $\mathrm{GaN}$. In the second part we will discuss the influence of strain in epitaxially lateral overgrowth $\mathrm{GaN}$ samples and in the final third part we use the $\mu$-Raman spectroscopy to investigate the incorporation of defects in GaN samples.

\section{Excitonic fine structure}

Typical emission spectra of thick GaN samples grown by hydrogen vapor phase deposition (HVPE) often shows narrow $A-, B$-, and $C$-exciton lines that could be used to investigate the excited states of free and bound excitons. The identification was made on the basis of reflection measurements [4] and supported faster by our magnetooptical experiments presented below. Figure 1 displays the low-temperature (LT) emission of polarized photons from a side face of a free-standing $300 \mu \mathrm{m}$ thick sample after band-to-band excitation in the range of the $n=1$ state of the $A$-exciton. In Fig. 1 , the detected light is polarized perpendicular (a) and parallel (b) to the $c$-axis. Both spectra are well fitted by a sum of two peaks and the underground from the neighboring weak $B$-exciton emission on the low-energy side and the stronger donor-bound $B$-exciton $\left(D^{0}, X_{B}\right)$. While the high-energy peak is seen at the same energy of $3.48000 \mathrm{eV}$ in both polarizations within our experimental accuracy a difference in the low-energy peak positions is observed between the perpendicular and parallel polarizations of Figs. 1a and b, respectively. In perpendicular polarization the measured peak position is $3.47904 \mathrm{eV}$ while in parallel polarization we find $3.47892 \mathrm{eV}$. We identify the latter value with the energy of the spin triplet state $\left(\Gamma_{6}\right)$ of the $A$-exciton and the former with that of the lower transverse branch of the spin singlet state $\left(\Gamma_{5 \mathrm{~T}}\right)$. Thus, the energy difference of $120 \pm 100 \mu \mathrm{eV}$ between the peaks corresponds to the splitting of these states caused by the exchange interaction between electron and hole in the $\Gamma_{5 \mathrm{~T}}$ state [5]. We assign the peak energy of $3.48000 \mathrm{eV}$ seen in both spectra to the position of the longitudinal branch of the spin singlet exciton $\left(\Gamma_{5 \mathrm{~L}}\right)$. This gives a value for the LT splitting of the $\Gamma_{5}$ state of $1.0 \pm 0.1 \mathrm{meV}$. This value is in very good agreement with the analysis of the reflection data mentioned above [4]. 
The dependence of the peak positions on the magnetic field is shown in Figs. 2a and b for $H \| c$ and $H \perp c$, respectively. For $H \| c$ the analysis of the energy differences reveals that the twofold splittings of both $\Gamma_{5}$ and $\Gamma_{6}$ states each follow the expected linear behavior. Thus, the $g$-values of the electron and the hole for this configuration can be determined, and they are $g_{\mathrm{e}}^{\|}=2.3 \pm 0.2$ and $g_{\mathrm{h}}^{\|}=-1.3 \pm 0.2$. The diamagnetic shift of the $\Gamma_{6}$ states is $4.0 \pm 0.3 \mu \mathrm{eV} / \mathrm{T}^{2}$. It is

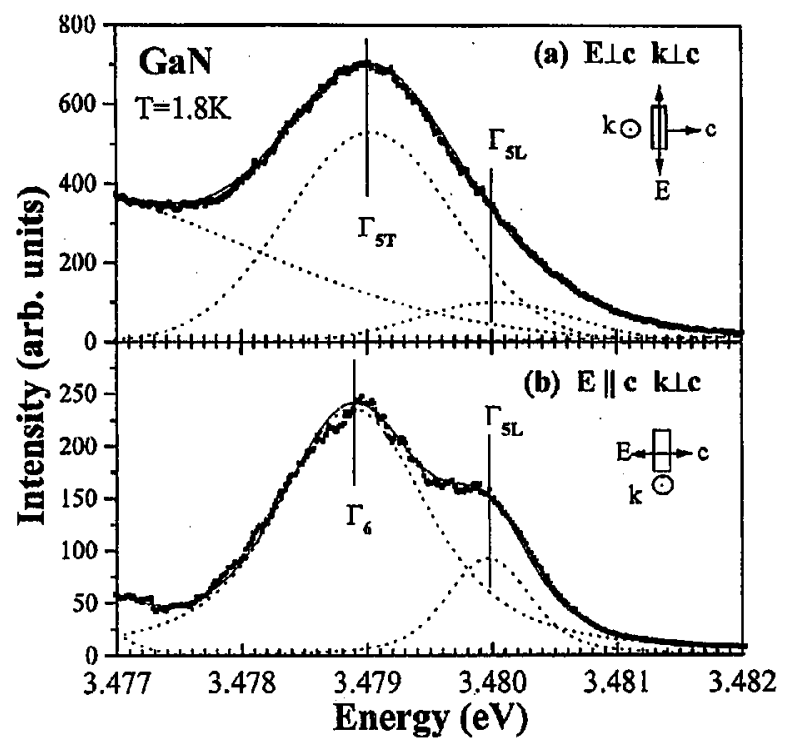

Fig. 1. Polarized photoluminescence (PL) from the state $n=1$ of the $A$-exciton. (a) $E \perp c$ and (b) $E \| c$.

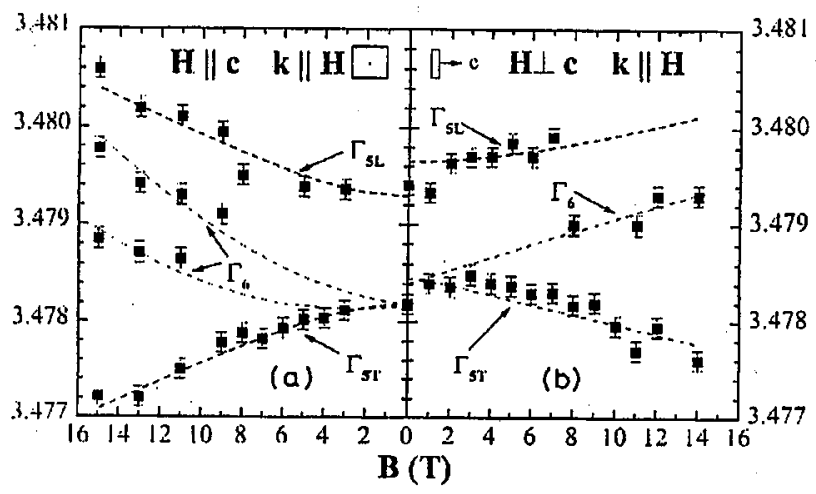

Fig. 2. Dependence of the energy levels of the state $n=1$ of the $A$-exciton on magnetic fields, (a) $\boldsymbol{H} \| \boldsymbol{c}$ and (b) $\boldsymbol{H} \perp \boldsymbol{c}$. 
interesting to note that the expected nonlinear splitting at low fields between the longitudinal and transverse $\Gamma_{5}$ states is indeed observed. It could not be observed in, e.g., CdS due to the large zero-field LT splitting of $2.1 \mathrm{meV}$ which is large enough to quench this mixing [6,7]. This point will be further discussed below. The observation of the mixing of these states is a very strong evidence for the correctness of our assignment of the respective emission lines and the value of the LT splitting determined in the zero-field PL spectra.

For $H \perp c$ one expects a mixing between $\Gamma_{5}$ and $\Gamma_{6}$ superimposed on the twofold spin splitting of each state. Due to the weakness of the emissions on the high-energy side of the $\Gamma_{5 \mathrm{~T}}$ in this configuration we can follow the $\Gamma_{5 \mathrm{~L}}$ only up to $8 \mathrm{~T}$. From there on a weak shoulder at lower energies due to emission from the $\Gamma_{6}$ state can be observed from which a resolution of the splitting in this state is not possible. From the splitting of the $\Gamma_{5 \mathrm{~T}}$ and $\Gamma_{6}$ states at higher fields the conduction band $g$-value is determined for this configuration and amounts to $2.0 \pm 0.2$, in good agreement with the results of other studies [8-10].

To summarize the results of this section, using polarized PL and magnetoluminescence we were able to detect for the first time the complete fine structure of the $n=1$ state of the $A$-exciton and its behavior in magnetic fields parallel and perpendicular to the $c$-axis.

\section{Selective epitaxial growth of GaN}

The selective epitaxial growth of GaN was performed on a $2 \mu \mathrm{m}$ thick $\mathrm{GaN}$ (0001) layer grown on a sapphire (on axis $\alpha-\mathrm{Al}_{2} \mathrm{O}_{3}$ (1120)) substrate using an AlN buffer layer. A $50-60 \mathrm{~nm}$ thick $\mathrm{SiO}_{2}$ mask was patterned by photolithography and subsequent etching to form hexagonal windows. The pattern consists of three periodic triangular lattices of hexagons having a width of $5 \mu \mathrm{m}$ and lateral distances of $10 \mu \mathrm{m}, 20 \mu \mathrm{m}$, and $40 \mu \mathrm{m}$, respectively. Finally, GaN was selectively grown on top, creating the periodic arrays of hexagonal shaped pyramids covered by the six $\{1101\}$ pyramidal facets. No $\mathrm{GaN}$ is deposited on the $\mathrm{SiO}_{2}$ mask. Growth details have been given elsewhere [11, 12].

The microscopical optical characterization was performed using spatially resolved cathodoluminescence (CL) and micro-Raman spectroscopy.

The spatially integrated low temperature $(5 \mathrm{~K}) \mathrm{CL}$ spectrum is shown in Fig. 3, together with local CL spectra obtained from the flat $2 \mu \mathrm{m}$ GaN layer between the masked pyramids, as well as the spectrum originating from the pyramids, respectively. Beside a narrow luminescence peak at $\lambda_{1}=355.0 \mathrm{~nm}\left(E_{1}=3.492 \mathrm{eV}\right)$ with a FWHM $=8 \mathrm{meV}$, stemming from the $2 \mu \mathrm{m}$ thick GaN layer below the $\mathrm{SiO}_{2}$ mask, an additional broad luminescence band is observed as a weak shoulder in the integral spectrum and is identified in the local CL as originating from the pyramids. This band is centered around $\lambda_{\mathrm{p}}=357.1 \mathrm{~nm}$ (i.e. $E_{\mathrm{p}}=3.471 \mathrm{eV}$ ) and is very broad with a FWHM $=52 \mathrm{meV}$, which indicates a local band gap fluctuation within the pyramid. This band-gap gradient from the base to the top of the GaN pyramids can be directly visualized through monochromatic CL images. Starting with an emission wavelength of $355.6 \mathrm{~nm}$ at the very top and at the edges of the pyramid, the whole $80 \%$ of the pyramid shows bright CL when $\lambda$ approaches $357.1 \mathrm{~nm}(3.471 \mathrm{eV})$, i.e., the emission of $\left(D^{0}, X\right)$ for un- 


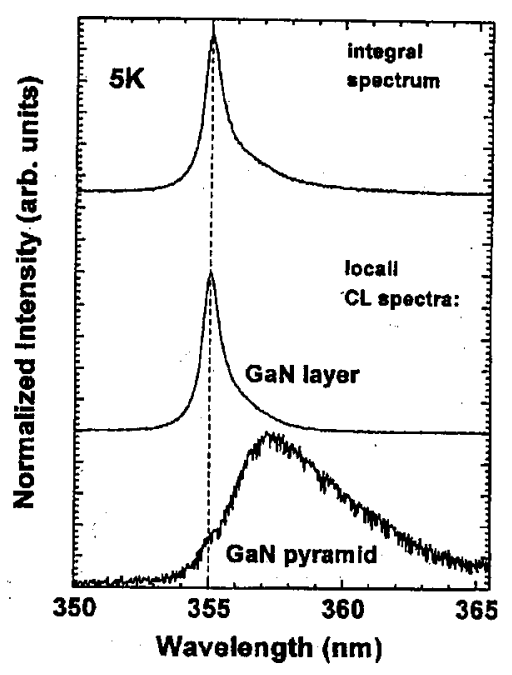

Fig. 3. Local spot-mode CL spectra taken at positions on the GaN pyramid and in between pyramids, compared with a spatially integrated CL spectrum.

strained or completely strain-relaxed GaN [13]. In addition, a strongly red-shifted CL with $\lambda>360.6 \mathrm{~nm}$ is emitted from the base area of the pyramid. A set of local CL spectra recorded at different positions along a $\{1101\}$ facet from the bottom to the top of a pyramid, plotted in Fig. 4, strongly confirms this observation. The different emission energies reveal the compressive strain of the $2 \mu \mathrm{m}$ thick GaN layer in contrast to the gradual relaxation of strain along the pyramid. From spatially resolved micro-Raman measurements performed at a position between the pyramids as well as focused on top of the pyramids, a compressive biaxial stress of $\sigma_{a}=0.95 \mathrm{GPa}$ (taking into account the best known value of $4.2 \pm 0.3 \mathrm{~cm}^{-1} \mathrm{GPa}^{-1}$ for the $E_{2}$ (high) Raman mode [14]) was found for the $2 \mu \mathrm{m}$ $\mathrm{GaN}$ layer whereas no stress is found when averaging over the pyramids. Thus, the CL results are in perfect quantitative agreement with the strain distribution obtained by Raman microscopy.

The stress distribution inside the pyramids and the $2 \mu \mathrm{m}$ connecting base layer has been investigated by elasticity theory through simulation by the finite-element method. The ingredients for the elasticity theory are the mismatch between GaN and the substrate and a set of elasticity constants. In the case of epitaxial growth the mismatch would have been given by the difference in the lattice constants. Our measurements seem to reflect a strong remnant of the epitaxial character of the material, in spite of dislocations and impurities in the sample. We were therefore encouraged to incorporate these uncertainties into a new mismatch parameter $\varepsilon_{\alpha}$ between the sample and the sapphire substrate and buffer, and continue to use epitaxial elasticity constants. The value of $\varepsilon_{\alpha}$ was adjusted to produce the biaxial stress $\sigma_{x x}$ observed in the connecting base layer.

Elasticity constants for epitaxial GaN from various authors have been tabulated in Ref. [14]. Our simulations showed that there was no qualitative difference 


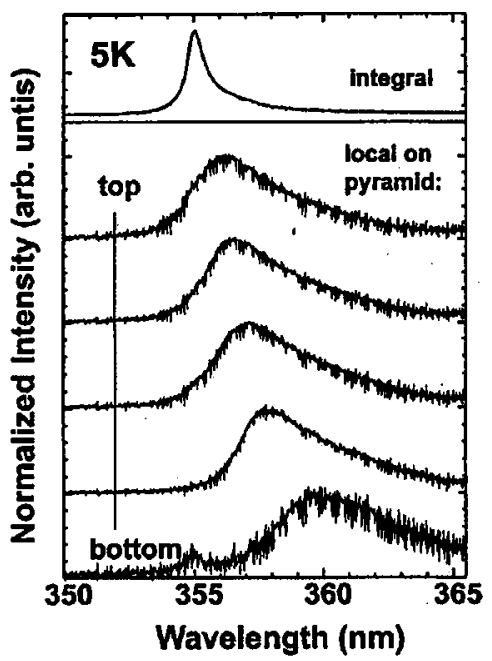

Fig. 4. Set of monochromatic CL images recorded at $T=5 \mathrm{~K}$.

in the stress distributions resulting from different sets of elasticity constants. $\varepsilon_{\alpha}$ was first adjusted to produce $\sigma_{x x}=1 \mathrm{GPa}$ for a uniform film. We obtained $\varepsilon_{\alpha}=0.2 \%$. As subsequent simulations demonstrated, the same $\varepsilon_{\alpha}$ led to $\sigma_{x x} \approx \sigma_{y y} \approx 1 \mathrm{GPa}$ in large parts of the connecting base layer even in the presence of pyramids. For this calculation, the lateral separation of the pyramids was $10 \mu \mathrm{m}$, the width of the hexagon was $6.66 \mu \mathrm{m}$, and the height of the pyramid was $10 \mu \mathrm{m}$. Due to rotation and mirror symmetries of the hexagon, simulation of only $1 / 12$ of the materials is necessary. A closer examination of the stresses along the sides of the pyramid revealed that the stress free volume extends $3 / 4$ of the way down from the top of the hexagon.

In Ref. [14] the stiffness matrix elements for the hexagonal GaN have been approximated by Young's moduli and Poisson ratios using Voigt averaging, i.e., elasticity constants for isotropic materials. The conclusions of our simulation remain the same, had we used the modified constants for isotropic materials. Moreover, combining the elasticity constants, whether for hexagonal or modified isotropic materials; from various authors listed in Ref. [14] and our mismatch parameter $\varepsilon_{\alpha}$, the biaxial stresses $\sigma_{x x} \approx 1 \mathrm{GPa}$ in all cases.

\section{Defect phonons in GaN}

Low-temperature Raman spectra of GaN films grown epitaxially on GaAs show, apart from the host lattice modes, a series of sharp lines in the low-energy spectral region. The intensity of these lines decreases nearly exponentially with increasing temperature and the lines disappear at room temperature. Since a vibrational Raman-scattering process should exhibit the opposite temperature behavior, i.e., an increase with increasing temperature [15], an electronic origin of these Raman lines seemed to be very likely. Ramsteiner et al. [16] reported on the observation of four such lines and interpreted these as electronic Raman transi- 
tions in a shallow donor in GaN. However, our measurements reveal the occurrence of additional lines which do not fit this model [17]. In contrast, in this work we present evidence that all lines are of vibrational, not of electronic, origin and we show that the responsible defects are related to the incorporation of As impurities into the $\mathrm{GaN}$ layers.

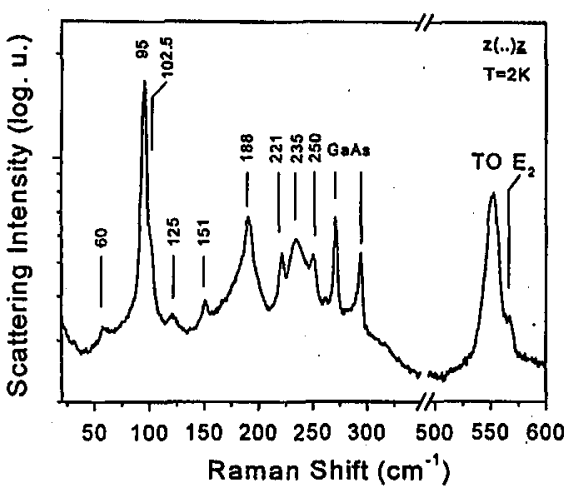

Fig. 5. Overview of the defect modes in GaN grown on GaAs. The spectrum was taken in $z(.)-$.$z configuration at 2 \mathrm{~K}$ with excitation at $514.5 \mathrm{~nm}(2.41 \mathrm{eV})$.

Figure 5 gives an overview of the phonon lines investigated in this paper. It shows a typical low-temperature Raman spectrum of a GaN layer grown on GaAs excited at $514.5 \mathrm{~nm}(2.41 \mathrm{eV}$ ). Apart from the host phonon modes of GaN (cubic TO and hexagonal $E_{2}($ high)) and GaAs (TO and LO) the low-energy part of the spectrum consists of several sharp lines, which are even more intense than the host Raman modes. We found in total at least 10 of these lines. The most intense lines are located at $95,150,190,220,235$, and $250 \mathrm{~cm}^{-1}$.

At first glance, considering the energy positions, one tends to assign these lines to second-order Raman scattering or to disorder-activated scattering either in the GaN layer or in the substrate. But the comparison with second-order Raman spectra of cubic and hexagonal GaN [18] as well as of GaAs [19] and the consideration of the phonon dispersions [18] show that these low-energy lines cannot stem from the GaN or GaAs host lattices. The low-energy parts of the second-order Raman spectra of GaN are dominated by structures at 314 and $415 \mathrm{~cm}^{-1}$. The lowest flat-running phonon branches which lead to a high phonon density of states and thus should be seen in disorder-activated scattering are located at around $150 \mathrm{~cm}^{-1}$. Consequently, the lines in question can only stem from defects built in the host material. These defects become visible in the Raman spectra either due to their vibronic or electronic excitations. For reasons of identification we will call these lines in the following defect lines. To scrutinize that they are really connected to the GaN layer and not to the substrate we also performed spatially-resolved micro-Raman measurements as well as measurements on series of samples with varying thicknesses. Both measurements confirm that the lines occur only in the GaN films. A line scan over the cross-section of a GaN/GaAs interface demon- 
strates that the intensities of the defect lines follow that of the GaN TO phonon mode, proving that they stem from the GaN layer not from the substrate.

In the following we will now focus on the question of the scattering mechanism and then turn to the identification of the defects involved in the scattering process. The defect lines weaken with increasing temperature and nearly disappear at $240 \mathrm{~K}$. The intensity variation with temperature $I(T)$ can be fitted by the following formula [16]:

$$
I(T)=A /\left[1+C \exp \left(-E_{\text {act }} / k T\right)\right],
$$

which gives us an activation energy $E_{\text {act }}$ of each line. The parameters $A$ and $C$ are temperature independent. We found activation energies in the range from 10 to $60 \mathrm{meV}$ depending on line and sample. This temperature behavior confirms the exclusion of host-lattice vibrations. Since defect lines exhibit the opposite temperature behavior, keeping in mind that vibrational Raman scattering from in-built defects should not follow the observed temperature behavior. Therefore, does this behavior already show that the defect lines are caused by electronic excitations of the defects in which the thermal occupation of the involved electronic states leads to the peculiar temperature dependence?

To answer this question one has to consider first which vibronic excitations of defects may be possible. One distinguishes between local modes, gap modes, and resonant (band) modes [20,21]. If the impurities built in the host lattice is of lower mass than the host atoms, one can observe local modes with frequencies higher than the phonon dispersion of the host lattice. This is obviously not the case here. Also gap modes can be ruled out. The defect lines are located in the acoustic region of the GaN phonon dispersion where no gap in the phonon density of states exists [18]. Under normal circumstance, those defect modes, which are degenerate with acoustic vibrations of the host material, should not be visible because motion relevant to the defect will rapidly degenerate into motion of the surrounding lattice, providing no discrete phonon states. Those modes can only be excited under resonant conditions (resonant (band) modes). Indeed, our defect lines exhibit a strong resonance behavior. They are excitable only in the region of the yellow luminescence between 2.0 and $2.5 \mathrm{eV}$ as can be seen from the Raman spectra taken with different excitation energies. It is thus conceivable that the lines are caused by vibronic excitations of the incorporated defects with only the resonance process having the observed temperature dependence. The scattering process would then have an indirect temperature dependence in contrast to the case of electronic Raman scattering in which the temperature dependence is caused directly by the thermal occupation of the involved electronic states.

Which electronic Raman processes could be possible? To explain the low energies of the lines only inner transitions in a shallow defect need to be considered. Acceptor transitions, as for example found in GaP [22], $\mathrm{ZnTe}$ [23], or $\mathrm{ZnSe}$ [24] are unlikely because our samples were all $n$-type and thus all acceptor states were occupied. In addition, the corresponding transition energies would be too large to fit the experimental findings, thus remaining only inner transitions in shallow donors. The latter also allow a straight forward explanation of the thermalization although optically determined activation energies do not need to be the same as 
those found in electrical measurements. However, in the case of inner transitions in shallow donors one would expect broader lines and an influence of the free carrier concentration which we could not observe in our samples [25, 26]. The high impurity concentration should lead to the formation of impurity bands resulting in a strong broadening of the lines. In Ref. [16] four of the lines at 189, 237, 151, and $217 \mathrm{~cm}^{-1}$ were attributed to an electronic Raman process in which electrons were excited from a deep acceptor level into the excited states of a shallow hydrogen-like donor. One pair of the lines at 189 and $237 \mathrm{~cm}^{-1}$ were interpreted as the $1 S-2 S$ and $1 S-3 S$ inner transitions in cubic $\mathrm{GaN}$ and the other pair should belong . $د$ the same transitions in the hexagonal modification of GaN, which was built in their samples as a minority phase. As we have already commented, our observation of additional lines with similar temperature and resonance behavior and thus similar origin contradicts this model [17].

In order to clarify the scattering mechanism we performed magnetic-field and pressure-dependent measurements on our samples. The intention was to observe characteristic shiftings and/or splittings of the lines in the case of an electronic Raman scattering process. Besides, pressure-dependent measurements allow us to tune continuously the band gap of the material investigated and thus to get further information on the resonance process. In our magnetic-field observation up to fields of $15 \mathrm{~T}$ we observe no shifting and splitting of the defect lines. This indicates that the defect lines are due to vibrational Raman scattering processes. Because the lines are not affected by an external magnetic field, inner electronic transitions in defects are very unlikely.

Similar to the study of the magnetic-field behavior, measuring the splitting/shifting of the lines in question under high hydrostatic pressure gives us more detailed information about the scattering mechanism and about the responsible defects.

Raman spectra taken from a GaN/GaAs sample with increasing hydrostatic pressure up to $6.1 \mathrm{GPa}$ exhibit no shift and no splitting of the defect lines. From the constancy of the line positions inner transitions in a shallow defect can be excluded. Applying hydrostatic pressure increases the band gap energy leading to an increase in the effective mass of the defect. Consequently, the energies of inner defect transitions also increase with applied pressure and one would expect a shifting of the Raman lines.

In addition, we investigated the effect of pressure on the temperature and on the resonance behavior. Our measurements reveal that neither changes significantly. We compare two low-temperature Raman spectra taken at $3 \mathrm{GPa}$ exciting at $514.5 \mathrm{~nm}$ and at $488 \mathrm{~nm}$. Although a pressure of $3 \mathrm{GPa}$ was applied to the sample the defect modes are still much more intense when exciting at $514.5 \mathrm{~nm}$ than at $488 \mathrm{~nm}$. This result has an important consequence for the involved resonance states. At first glance a link to those electronic states which are responsible for the yellow luminescence seemed to be likely because the Raman excitation profile of the defect lines is located in the same energy region. Although in this case one would already have to consider that the Raman excitation profile should not follow the luminescence but instead the luminescence excitation spectrum, because only the latter one reflects the energy positions of the involved electronic 
states. Consequently, one would expect an increase in the resonance profile in the high-energy region which is not found experimentally. Our high-pressure measurements confirm these doubts. They demonstrate that the resonance states of the defect modes can neither belong to the yellow luminescence nor to any states related to the conduction band. In both cases one would expect a shifting of the resonance profile of about $120 \mathrm{meV}$, based on the experimentally found pressure coefficient of $40 \mathrm{meV} / \mathrm{GPa}$ [27, 28]. If a shallow defect or the conduction band would be involved in the resonance process one would expect the inverse intensity distribution. The defect lines should be more intense in the spectrum excited at $488 \mathrm{~nm}$ than in the spectrum excited at $514.5 \mathrm{~nm}$. In conclusion, no shallow states can be involved in the resonance process.

After clarifying the principle scattering mechanism we now turn to the identification of the responsible defects. We performed measurements on several series of undoped $\mathrm{GaN}$ layers grown on different substrates and by different growth techniques (MBE, MOCVD, HVPE). The defect lines were present only in those spectra which were taken from GaN layers deposited on GaAs substrates. We thus assumed that the incorporation of arsenic from the substrate into the layer might form defects which are responsible for the lines in question. To prove our assumption we measured GaN layers grown on sapphire which were intentionally doped with arsenic. Secondary-ion mass spectroscopy (SIMS) revealed an arsenic concentration of around $10^{18} \mathrm{~cm}^{-3}$ in this layer, which is in the same range of the arsenic concentration in our GaN/GaAs samples [to be published elsewhere]. A spectrum taken from one of these samples after excitation at $514.5 \mathrm{~nm}$ is shown in Fig. 6 . The strongest lines are located at 96,178 , and $235 \mathrm{~cm}^{-1}$. The broad background below $300 \mathrm{~cm}^{-1}$ is due to disorder-induced scattering and can also be observed in GaN/GaAs and in ion-damaged GaN. The energy positions are in very good agreement with the lines known from the GaN/GaAs samples. The lines also exhibit the same temperature behavior. They decrease nearly exponentially in intensity with increasing temperature. The activation energies are between 10 and $60 \mathrm{meV}$, i.e., in the same range as the lines known from the GaN/GaAs samples. We are thus certain that these lines have the same origin and that the incorporation of arsenic into the GaN layer leads to the responsible defects. But it remains unclear whether arsenic is directly involved, i.e., whether the defect contains arsenic, or arsenic acts only as a catalyst.

We also investigated $\mathrm{Si}-, \mathrm{C}$-, and $\mathrm{Mg}$-doped $\mathrm{GaN}$ samples grown on sapphire, but we could not observe any of the defect lines. It is noteworthy that the resonance behavior differs slightly from the one in $\mathrm{GaN} / \mathrm{GaAs}$, in that the narrower lines at 95 and $105 \mathrm{~cm}^{-1}$ are even more intense when exciting in the red spectral region.

Summarizing our results, the low-energy Raman lines cannot stem from the host GaN or substrate lattice. Neither disorder-activated scattering nor second-order Raman scattering can explain their energy positions and their small line widths. We thus related these lines to excitations of in-built defects. This is confirmed by the unusual temperature behavior. As the comparison of several series of samples showed, the lines occur only in samples grown on GaAs. But the lines can also be produced in GaN layers grown on sapphire when doping these layers with arsenic clearly shows that the lines are associated with arsenic-related de- 


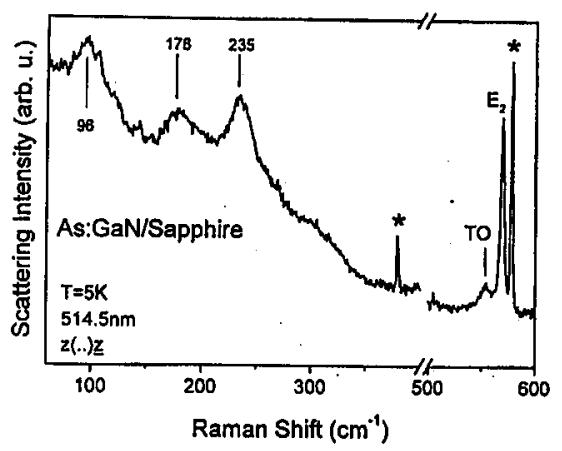

Fig. 6. Low-temperature Raman spectrum of an As-doped GaN layer grown on sapphire. Three of the defect modes at 96,178 , and $235 \mathrm{~cm}^{-1}$ occur. The asterisks denote lines of the sapphire substrate. The temperature was $5 \mathrm{~K}$, the excitation wavelength was $514.5 \mathrm{~nm}(2.41 \mathrm{eV})$.

fects. The lines decrease drastically in intensity with increasing temperature and vanish at room temperature excluding normal vibrational Raman scattering as their origin. This temperature behavior can be explained by two opposite types of defect-scattering mechanisms: Either the lines originate from electronic Raman transitions in which the thermal occupation of the involved electronic defect states serves for the temperature dependence or the lines are caused by resonant vibrational Raman scattering (resonant modes) in which only the resonance process exhibits the thermalization. Our magnetic field-dependent and pressure-dependent Raman measurements clarified this point. Because neither splitting nor shifting was observed in these measurements inner electronic transitions of defects are very unlikely. Thus, our results strongly indicate a resonant vibrational Raman process, with only the resonance having the observed temperature behavior.

In conclusion the temperature behavior indicated an electronic Raman scattering process, our pressure- and magnetic-field dependent measurements definitely show that they are of vibrational origin, most likely resonant modes of arsenic complexes in the GaN layers.

\section{Acknowledgments}

The authors are indebted to all growth teams, especially K. Hiramatsu for the HVPE GaN samples, D.J. As, D. Schikora, B. Schöttker for providing the GaN/GaAs samples and S. Einfeldt and D. Hommel for the doping series of GaN on sapphire. We also thank K. Syassen for the help with the pressure measurements and I. Loa for the assistance during the magnetic-field dependent measurements. 


\section{References}

[1] J.J. Hopfield, D.G. Thomas, Phys. Rev. 122, 35 (1961).

[2] L. Eckey, L. Podlowski, A. Göldner, A. Hoffmann, I. Broser, B.K. Meyer, D. Volm, T. Streibl, K. Hiramatsu, T. Detchprohm, H. Amano, I. Akasaki, in: Int. Conf. Silicon Carbide and Related Materials, Kyoto 1995, Eds. S. Nakashima, H. Matsunami, S. Yoshida, H. Harima, in series Inst. Phys. Conf. Ser., Vol. 142, Institute of Physics Publ., Bristol 1996, Ch. 5, p. 943.

[3] M. Steube, K. Reimann, D. Fröhlich, S.J. Clarke, Appl. Phys. Lett. 71, 948 (1997).

[4] A. Hoffmann, Adv. in Solid State Phys. (Festkörperprobleme) 36, 33 (1996).

[5] K. Cho, Phys. Rev. B 10, 4463 (1976).

[6] I. Broser, M. Rosenzweig, Phys. Rev. B 22, 2000 (1980).

[7] G. Blattner, G. Kurtze, G. Schmieder, C. Klingshirn, Phys. Rev. B 25, 7413 (1982).

[8] W.E. Carlos, J.A. Freitas, Jr., M. Asif Khan, D.T. Olson, J.N. Kuznia, Phys. Rev. $B$ 48, 17878 (1993).

[9] E.R. Glaser, T.A. Kennedy, H.C. Crookham, J.A. Freitas, Jr., M. Asif Khan, D.T. Olson, J.N. Kuznia, Appl. Phys. Lett. 63, 2673 (1993).

[10] M. Kunzer, U. Kaufmann, K: Maier, J. Schneider, N. Herres, I. Akasaki, H. Amano, Mater. Sci. Forum 143-147, 87 (1994).

[11] K. Hiramatsu, S. Kitamura, N. Sawaki, Mater. Res. Soc. Symp. Proc. 395, 267 (1996).

[12] S. Kitamura, K. Hiramatsu, N. Sawaki, Jpn. J. Appl. Phys. 34, L1184 (1995).

[13] H. Siegle, A. Hoffmann, L. Eckey, C. Thomsen, J. Christen, F. Bertram, D. Schmidt, D. Rudloff, K. Hiramatsu, Appl. Phys. Lett. 71, 2490 (1997).

[14] C. Kisielowski, J. Krueger, S. Ruvimov, T. Suski, J.W. Ager, E. Jones, Z. Liliental-Weber, M. Rubin, E.K. Weber, M.D. Bremser, R.F. Davis, Phys. Rev. $B$ 54, 17745 (1996).

[15] M. Cardona, in: Light Scattering in Solids II, Eds. M. Cardona, G. Güntherodt, in series Topics Appl. Phys., Vol. 50, Springer, Berlin 1982, p. 19ff.

[16] M. Ramsteiner, J. Menninger, O. Brandt, H. Yang, K.H. Ploog, Appl. Phys. Lett. 69, 1276 (1996).

[17] H. Siegle, I. Loa, P. Thurian, L. Eckey, A. Hoffmann, I. Broser, C. Thomsen, Appl. Phys. Lett. 70, 909 (1997).

[18] H. Siegle, G. Kaczmarczyk, L. Filippidis, A.P. Litvinchuk, A. Hoffmann, C. Thomsen, Phys. Rev. B 55, 7000 (1997).

[19] R. Tromuler, M. Cardona, Phys. Rev. B 17, 1865 (1978).

[20] W. Hayes, R. Loudon, Scattering of Light by Crystals, Wiley \& Sons, New York 1978.

[21] A.S. Barker, Jr., A.J. Sievers, Rev. Mod. Phys. 47, Suppl. 2, S1 (1975).

[22] D.D. Manchon, Jr., P.J. Dean, in: Proc. 10th Int. Conf. Phys. Semicond., Eds. S.P.S. Keller, J.C. Hensel, F. Stern, USAEC Division of Technical Information Extension, Oak Ridge (Tenn.) 1970, p. 760.

[23] S. Nakashima, T. Hattori, P.E. Simmonds, E. Amzallag, Phys. Rev. B 19, 3045 (1979). 
[24] D.J. Olego, J. Petruzzello, T. Marshall, D. Cammack, J. Cryst. Growth 117, 324 (1992).

[25] M. Holtz, R. Zallen, O. Brafman, Phys. Rev. B 37, 2737 (1988); ibid. 38, 6087 (1988).

[26] K. Jain, Phys. Rev. B 13, 5448 (1976).

[27] W. Shan, T.J. Schmidt, R.J. Hauenstein, J.J. Song, B. Goldenberg, Appl. Phys. Lett. 66, 3492 (1995).

[28] T. Suski, P. Perlin, H. Teisseyre, M. Leszczyński, I. Grzegory, J. Jun, M. Boćkowski, S. Porowski, T.D. Moustakas, Appl. Phys. Lett. 67, 2188 (1995). 